\title{
An Overview of MEMS Biosensor
}

\author{
Md Saeed Hasan Joarder ${ }^{1}$, Dr. Badrul Hisham Bin Ahmad ${ }^{2}$ \\ ${ }^{1-2}$ Faculty of Electronic and Computer engineering, University Teknikal Malaysia Melaka, Malaysia, \\ ${ }^{1}$ saeed.ru.54@gmail.com, ${ }^{2}$ badrulhisham@utem.edu.my
}

\begin{abstract}
Biotechnology is rapidly advancing and gaining a lot of attention to the researcher due to its advantages, and biosensor is the key to advancing in biotechnology. Now a day's MEMS-based sensors are gaining a lot of attention due to its high sensitivity. MEMS-based sensors have a high potential for detecting the different types of biomolecules. It uses simple methods to analyze the molecule. These sensors are now used in many biomedical instruments to diagnose different types of diseases. Here we will discuss the different technology used by the MEMS-based sensor to determine different molecules.
\end{abstract}

Key words: Biosensor, Biomolecule, BAW, Electrospinning, MEMS, SAW,

\section{INTRODUCTION}

Nowadays, sensors are using almost all kinds of electronic equipment. Its uses in IoT devices, wireless communication, medical instrument, etc. Sensors are using in the vehicle to reduce road accidents and also it can be used to build smart places like a smart car parking area.[1][2] Miniature circuits now available at a much lower cost with higher efficiency, and the MEMS sensor is helping convert large sensors to a small one. Almost all kinds of biomedical equipment used a sensor for different purposes. Biosensors are critical devices for various biomedical equipment. In these sensors, Biomolecular collaboration is used to identify the responses. These sensors generally have a receptor whose mechanical or chemical or electrical properties changed when it came under the contact of different biomolecule types such as antibodies, antigens, enzymes, etc. A biosensor responses according to the biological input to the sensor, and the sensor output electrical, mechanical, optical, thermal properties changes according to the response. There are many types of biosensors available and used in equipment for virus detection, blood sugar detection, exhaled breath analysis, etc. It can also detect complex brain circuit/neural activity.[3] These biosensors generally consist of a sensing layer and a transducer. This transducer gets the sensing layer's response, and it detects a specific molecule with quantity.
A Microelectromechanical system is one of the most useful systems to manufacture biosensors. With this system, the desired biosensor can be produced at a low cost, small package for various bioanalytical devices.[4] MEMS technology can be used to fabricate biosensor in a batch, and the sensor can be aligned in the array, for which similar results can be obtained from the array of sensors. Thus a more accurate result can be obtained. The MEMS-based sensor utilizes microchannels, cavity, microcantilever, etc.[5][6] There is some infectious disease whose early detection is quite tricky and very expensive and difficult to diagnose. A MEMS-based biosensor can be used to eliminate all those difficulties in diagnosis a disease. It has so many advantages as it can be used in portable devices that do not need any complicated procedure to detect any disease. Those devices need minimal knowledge to operate and can be reused so often that disease can be rapidly detected. MEMS-based sensors are quite useful because of sound thermal isolation, vibration isolation, [7] fast result, and low power consumption. Several types of technology are used in MEMS-based sensors such as surface acoustics wave, Bulk acoustics wave, electrochemical, electrospun, Thermal, optical, viscometric, etc.

\subsection{Surface acoustics wave Based MEMS sensor}

SAW biosensors are those whose output measurement depends on the propagation of surface acoustics wave and sensing material placed on the MEMS platform. This sensor can be resonant with a particular surface acoustics wave. These sensors appear to be very useful for biomedical applications for its sensitivity and perfect response time. It does not need any labeled molecule compared to other methods, such as enzyme-linked immune absorbent assay and radio-immunoassay. [8] It is the only technique that can detect biomolecule and fluid manipulation. [9] These sensors can be used to detect a lot of infectious diseases that need early detection. For point-of-care(POC) testing, this sensor-based device is beneficial as it does not need any complicated diagnostics procedure.[10] As the name depicted, acoustics wave propagates along the substrate's surface in surface acoustic wave sensors. When these sensors open to the particular molecule, then the resonant frequency of surface acoustics wave changes by which the substances of those molecules can be detected. SAW-based sensors have an interdigital Transducer and analytes placed between the two electrodes of the transducer. In these sensors, a voltage is 
applied to the piezoelectric material through the interdigital transducers electrode. Due to the piezoelectric effect, the material will show a mechanical deformation that causes an acoustic wave. Now, suppose biomolecules come under contact with that piezoelectric material. In that case, there is a change in the generated acoustics wave on that material and can shift the resonant frequency of that material. Such as if an aqua specimen is applied to the sensing layer, then the acoustics wave properties will change due to its viscosity of the liquid, and it can be seen that the acoustics wave velocity will be changed. Another electrode of the interdigital transducer can detect this change. By manipulating the shifting pattern of resonance frequency, a molecule can be detected. Typically sensors show the different properties for analytes mass, density, viscosity, velocity, etc.[11] Fig-1 shows a SAW sensor.

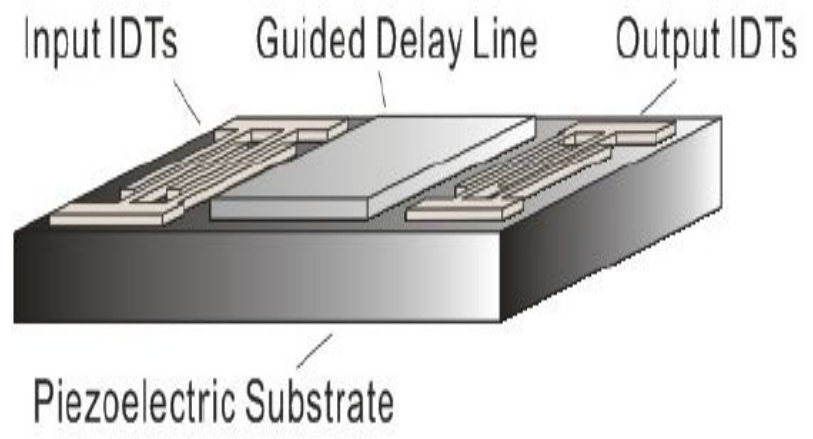

Figure 1: SAW sensor[8]

\subsection{Bulk acoustics wave based MEMS sensor}

In this sensor, Bulk acoustics wave(BAW) resonator is used. These sensors consist of a piezoelectric material and two electrodes. Gold, Platinum, Aluminum, Chromium are used for those electrodes. These two electrodes placed each side of the piezoelectric material. For BAW, mostly Aluminium nitride(AIN) or zinc oxide( $\mathrm{ZnO})$ is used as a piezoelectric material.[12] It has a higher quality factor as it has lower viscous losses.[13] Acoustics wave propagated through the piezoelectric material from one electrode to another when a voltage is applied to that electrode. The formation of this acoustic wave due to the electric field generated along the direction of the piezoelectric material's thickness. Now analyte is placed on the electrode, which is placed on the top side of the sensor. There is a change in the propagation of acoustics waves through the piezoelectric material. Generally, the change in acoustics waves occurred due to the mass loading effect. As the mass of the analytes changes, piezoelectric material resonant frequency also changes, and its frequency range few $\mathrm{MHz}$ to $10 \mathrm{GHz}$ and this frequency depends on the thickness of the piezoelectric material, which can be few micrometers to few hundred micrometers and lower the thickness of the piezoelectric material, higher the resonant frequency. The higher the resonance frequency of the resonator, the higher the sensitivity of the sensor. This sensor has to be placed in a soundproof environment during analytes analysis so that external sound could not interfere. This sensor can be fabricated using two types of resonator: Film Bulk acoustics resonator(FBAR) and solidly mounted resonators(SMR). On the SMR sensor's bottom side, an acoustics mirror is used to be confined to a particular acoustics waveform. These acoustics mirrors are a layer of different material which have different acoustics impedance properties. This mirror is called a Bragg mirror or Bragg reflector[14]. In Bragg mirror, high acoustic impedance material like Pt and low acoustics impedance AIN can be used in several layers. There is another method where an air cavity is created between resonator and substrate. A resonator's generated acoustics wave is reflected between the cavity surfaces. Its called film Bulk acoustics resonator(FBAR), shows in Fig-2.

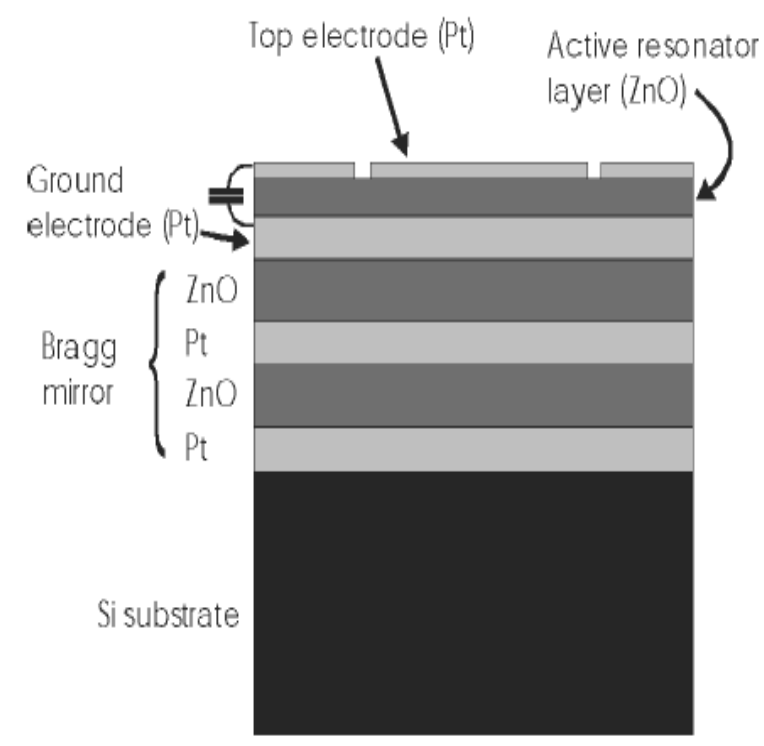

Figure 2: BAW Sensor[27]

\subsection{Electrochemical MEMS biosensor}

The electrochemical sensor uses electrochemistry to detect the biomolecule shown in Fig-3. Electrochemical biosensors have excellent sensitivity, good response time, and it has a very high potential for different biomedical applications for diagnostics. Mostly these sensors are very selective. The sensing technique is much more straightforward than other technologies and has less background current[15], less interference. Other techniques as optical and acoustics wave technique, have interference issues with light and sound. Different types of material are used for sensing material such as pyrolyzed carbon electrode, gold electrode. However, most of the time, carbon is used for the sensing electrode as it has excellent electrochemical stability. Carbon is also inexpensive and readily available. It can be seen that carbon also has superior biocompatibility [16], and it has excellent chemical, mechanical, electrical, and thermal properties. Its 
sensitivity can be increased by increasing the electrode surface, which can be achieved by decreasing the electrode's size. Graphene-based material, single-walled carbon nanotubes, multi-walled carbon nanotubes, glassy carbon, and nanoporous carbon are perfect for using an electrochemical sensor. It can be used to detect different types of viral diseases, enzymes. The viral disease can be detected by immobilizing different types of antibodies and capturing the antigen by which the electrical properties will change and show different current-voltage characteristics due to redox cycling when it's exposed to the analytes, which can be used to detect Hepatitis-A, Hepatitis-B, Hepatitis C, HIV, Nov, etc. Enzymes are also used to immobilize on the nanoporous carbon electrode to make the sensing layer. Thus Glucose of the sample can be measured, and diabetes can be detected[17].

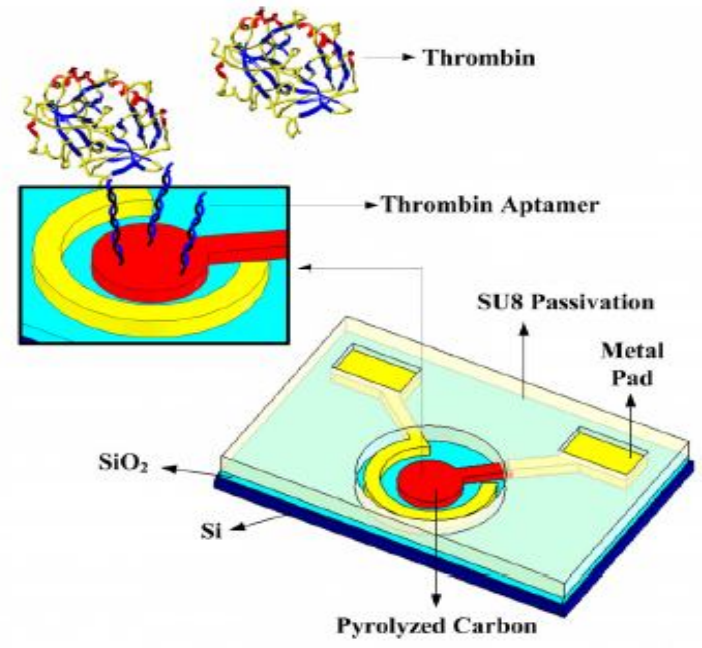

Figure 3: An electrochemical biosensor[15]

\subsection{Electrospinning biosensor}

Electrospinning biosensors can be created using nanofiber, nanowire, etc. It is seen that most of the biosensor sensing material fabricated on the substrate, and that's a drawback as the sensing materials all the side cannot be exposed to that biomolecule and that can reduce the efficiency of a biosensor but if the sensing material is a nanowire and that can be suspended then the maximum area of sensing layer exposed to the biomolecule. It will have excellent sensitivity and produce an excellent result. With the Electrospinning process's help, it is possible to fabricate biosensors whose sensing layer can be suspended. All sides of the sensing material can be exposed to the desired analyte. As sensing material is not attached to any other material, the parasitic effect will also be minimal. This method is not much complicated but cheap and can be fabricated quickly. Most of the time, the polymer used for nanowire, nanofiber, and nanotube due to its sensing response, chemical properties, and small footprint, but metal oxide, which has excellent biosensing properties, also can be used in here by coating the material on top of the polymer nanowire. The polymer can be used for absorption, adsorption, and viral filtration of biomolecular for biosensor.
Due to these properties, its current conduction changes, and a particular molecule can be detected.[18] Polymer nanowire fabricated on the MEMS platform. In this technique, a syringe is used, which has a pump also. The polymer solution is placed in that syringe, and then a high voltage is applied to that polymer solution, which makes the solution charged. A collector is placed in front of the syringe needle, and that collector is connected to the ground. When the high voltage is applied to the polymer, a jet of polymer flows from the syringe to the collector due to the electric field and jet flows to the electric field's direction. Thus nanowire or nanofiber formed.[19] Nanofibers produced here are few nanometers to few microns. Nanowire fabrication method shows in Fig-4.

(a)
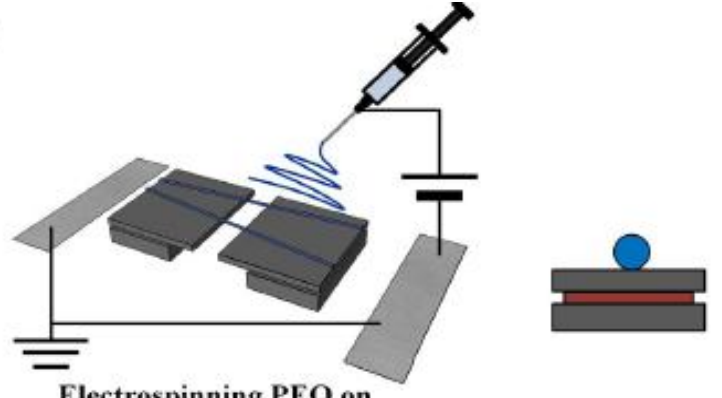

MEMS platform with micrometer gap

(b)
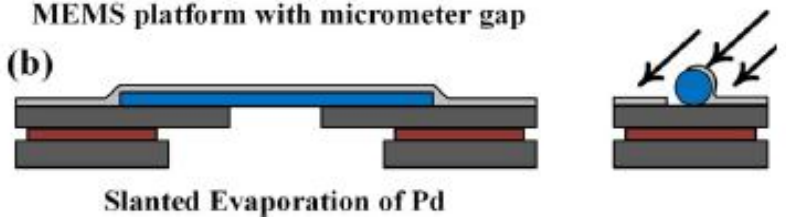

(c)
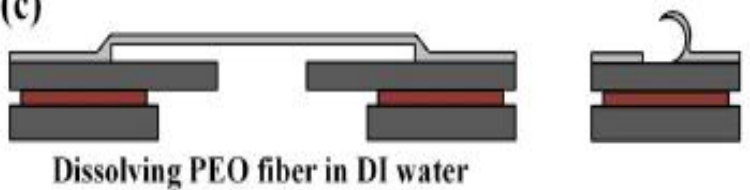

(d)
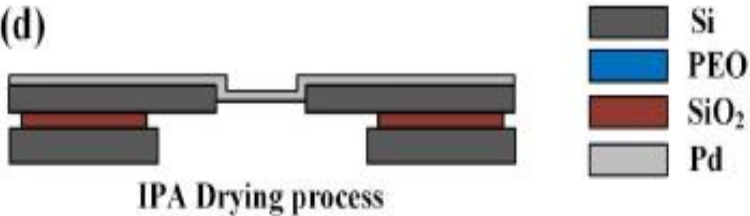

Figure 4: Fabrication process of the nanowire using electrospinning on MEMS platform. [28]

\subsection{Thermal MEMS-based Biosensor}

MEMS thermal biosensor analyze biomolecule by utilizing the measurement of thermal energy released during the biochemical reaction. This sensor mainly utilizes the heat produced by an enzymatic reaction. These sensors help detect an element without any complex cascaded reaction process used by other methods. Generally, thermistor and thermopile are used in this sensor.[20] In the thermistor process, change in the sensor's resistance is measured during the heat produced by analytes caused by some reaction. With the help of TCR of a thermistor, the bimolecular property can be analyzed. During the analysis, there may be some external interference that may affect the analysis. A reference 
characteristic of the thermistor is used to remove the external and internal interference. The thermistor signal needed some amplification as most of the signal is weak. Thermopile is very good compared to the thermostat, as it has a significantly higher sensitivity. Thermopiles can be made of metal or semiconductor, and its sensitivity can be increased by making the sensing material suspended by which thermal mass will be reduced. Thus the sensitivity increases, and it can detect nanojoule thermal changes. With the help of a thermal biosensor, solid, fluidic, and gaseous analytes can be analyzed. In this system, a thermopile is constructed where one side of the thermopile can be exposed to the analyte. The other side is exposed to a reference solution that exhibits temperature differences in thermopile. [21]As its sensing layer with analytes output changes respect to a reference solution, its background noise will be canceled. Due to the temperature difference, a voltage induced on the thermopile changes proportional to temperature differences. A heater is also used to get the desired temperature of analytes and reference samples, which also can be used for this sensor calibration. [22] In the MEMS-based sensor analytes, inject through the microchannel of the sensor. Fig-5 shows a Thermal MEMS-based sensor.

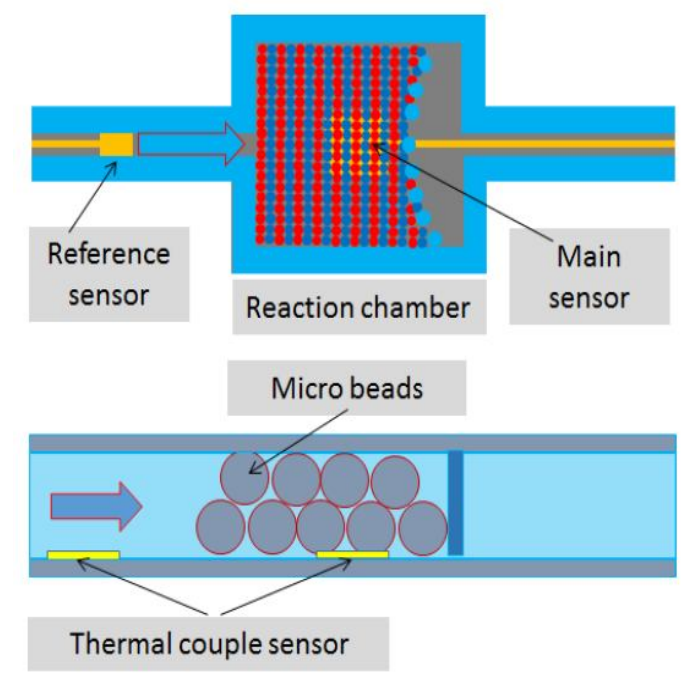

Figure 5: Schematic of the thermal biosensor[20]

\subsection{Optical MEMS biosensor}

An optical-based biosensor utilized the change of mechanical or electrical properties when it came under contact with biomolecules. Several methods to detect molecule by analyzing optical properties changes or sensing layer characteristics changes due to optical properties change. In the interferometric method, light is passed through the desired analytes, and some of the light reflects the source, which causes interference. The photosensor can detect this interference, and thus the properties of analytes are measured by analyzing the interference. There is another interferometric method in which the material used for the sensing layer is soft. When exposed to the analytes like an antigen, it will react with immobilizing antibody, and the surface of the sensing layer can be deformed. One side of the sensor has a photodetector, and the other side has a light source of a single wavelength. Due to the sensing layer's deformation, a photodetector can detect a change in wavelength, and the molecules can be detected.[23] Surface plasma resonance is one of the most popular ways to analyze biomolecule in an optical-based MEMS sensor. In this method, light is incident on metal like Au is used as a sensing layer, reflecting on the detector. Gold is used most of the time because of its bioconjugation. All metal has its resonance frequency, and this resonance frequency can be driven by light from an external source. When light strikes the metal on a particular angle, then the light is absorbed by the metal electron, and resonance occurs in the electron. In the reflected light, a portion of a waveband of light is absorbed by the sensing layer that is detected by a photodetector. The resonance electron is sensitive to the surrounding environment. When analytes come close to the sensing layer, the absorbed light band is shifted according to the analytes' nature, which can be used to identify biomolecule. A colorimetric biosensor is also had exquisite sensitivity, which also uses the principle of plasma.

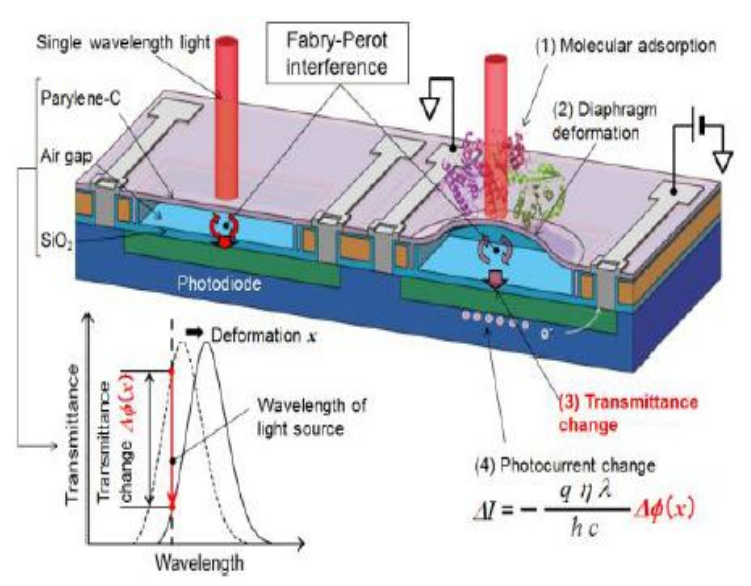

Figure 6: Schematic of the surface stress sensor based on a MEMS.[23]

In this method, molecules are detected by the reflection of a different color. In this method, the sensor reflects different colors when a light incident on the sensor and then the light incident on the sensor through the biomolecule and the wavelength of light changes, which shows a different color. By detecting those color changes, analytes can be identified [24]. Fig-6 shows an optical MEMS biosensor.

\subsection{Viscometric MEMS sensor}

As the name implies, the viscometric MEMS sensor utilizes the sample's viscosity properties. There is a decent amount of application in biomedical to use this type of sensor. This type of sensor can be used to measure concentration and type of sample. Most of the time, the sensor's mechanical properties are used to identify the sample. A microcantilever is fabricated in the MEMS sensor shown in Fig-7, which shows deformation when the sample is applied to that cantilever. 
Md Saeed Hasan Joarder et al., International Journal of Emerging Trends in Engineering Research, 8(9), September 2020, 6464 - 6469

[25] This cantilever can be fabricated in parallel, which can be worked as the capacitor plates. If any fluid sample is applied between that cantilever, it can work as a capacitor's dielectric material. Samples dielectric properties can change with the change of sample viscosity. If this capacitor is used as part of an LC circuit that can oscillate, it can be used to analyze the sample as sample properties change. The capacitance changes which affect the oscillation frequency and sample can be detected. In another method, the cantilever resonance frequency is measured. An external frequency equal to resonance frequency applied so that there is a vibration in the cantilever. This frequency of vibration is changed when an analyte is applied to the cantilever due to sample viscosity. This vibration can be measured using the optical lever method. [26] By the optical lever method, minimal displacement of objects can be measured. Vibrating frequency and amplitude reduces due to applied analytes. Thus measuring the amplitude, phase shift, vibrating frequency help to detect the sample property.

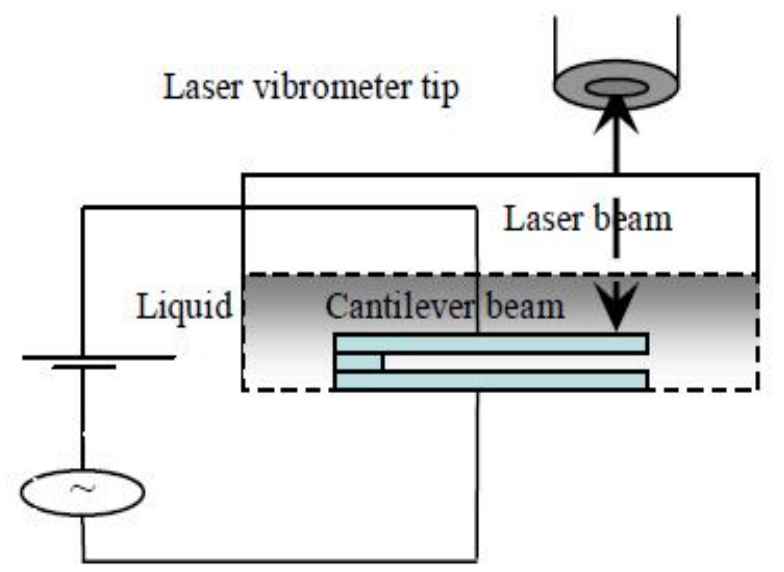

Fig-7: Viscosity sensor using microcantilever [25]

\section{CONCLUSION}

MEMS-based biosensors became an integral part of biomedical applications. Micromachining makes the system size significantly lower than the bulky, heavy system. It makes it possible to manufacture a device that can be used in point of care. Portable equipment can be easily manufactured. The cost of diagnosis of various diseases became significantly lower. Extensive research is going on this type of sensor, and it is making our life easy.

\section{ACKNOWLEDGEMENT}

The authors would like to thanks Universiti Teknikal Malaysia(UTeM) Melaka for providing all the supportive information and material for the work. We would also like to thank the Faculty of Electronics and Computer Engineering of UTeM and acknowledge their support. Lastly, thanks to all the referees, the lecturer who has helped to complete this paper.

\section{REFERENCES}

1. Vehicle Blind Spot Monitoring Phenomenon using Ultrasonic Sensor, Adnan, Hassan, Ab Wahab , Najib, Nasir, Universiti Teknikal Malaysia Melaka, Malaysia, IJETER 2020 .

2. MQTT_SN_QoS: an enhanced MQTT protocol for wireless sensor network in smart parking, Hicham Ouldzira, Ahmed Mouhsen, Mohamed Hassoun , Rachid Habachi , Mustafa Elalami, Departement of Electrical Engineering, Sciences and technology Faculty, Hassan 1st University Settat, Morocco, IJETER 2020

3. A new MEMS neural probe system integrated with push-pull microfluidic channels and biosensors for real-time monitoring of neurochemicals, Uikyu Chane, Hyoguen, Hyunjoo Jenny Lee, Jungpyo Lee, Nakwon Choi, Yi Jae Lee, SOO Hyun Lee, Jiwan Woo, Yakodol Cho, Eui-Sung Yoon, Hyun-Yong Yu, H-Joo Cho, IEEE 2016.

4. Detection of Hepatitis viruses (HBV, HAV, HCV)in serum using MEMS based Bio-Sensor, Nireekshith Yarraguntla, Naveena Tirumala, Syed Shameem, K. srinivasa rao, Second International Conference on Computing Methodologies and Communication (ICCMC 2018).

5. SMART BIOSENSOR FOR RAPID AND SIMULTANEOUS DETECTION OF WATERBORNE PATHOGENS IN TAP WATER, Sura A. Muhsin, Muthana Al-Amidie, Zahar Mlaji, Zhenyu Shen, Amjed Abdullah, Jiayu Liu,Ferris Dweik, Ibrahem Jasim, Majed El-Dweik, Shuping Zhang, and Mahmoud Almasri, Transducers 2019 - EUROSENSORS XXXIII Berlin, GERMANY, 23-27 June 2019

6. Frequency Profile Measurement System For Microcantilever-Array Based Gas Sensor, Maira Possas, Lionel Rousseau, Farbod Ghassemi Gaelle Lissorgues, Emmanuel Scorsone, Rafaa Manai, Philippe Bergonzo Diamond Sensors Laboratory, CEA LIST, 2015 Symposium on Design Test Integration and Packaging of MEMS and MOEMS.

7. Design and Simulation of MEMS Thermal and Vibration Isolator Based on PDMS Beam Arrays, Kaisi Xu, Ningli Zhu, Shan Cao, Weiguo Su, Wei Zhang, Yilong Hao, International Conference on Nano/Micro Engineered and Molecular Systems (NEMS), 2016.

8. Performance Optimization of Temperature Compensated Surface Acoustic Wave Biosensors, Shuangming Li, Yan Su, Ying Wan, Zheng Tang, 2013 Seventh International Conference on Sensing Technology.

9. Full-SAW Microfluidics-Based Lab-on-a-Chip for Biosensing MATTEO AGOSTINI, GINA GRECO, AND MARCO CECCHIN, IEEE 2019.

10. Microcavity assisted acoustic wave channeling can lead to high sensitivity and ultra-low power SAW sensors, Sina Koochakzadeh, Mandek Richardson and Venkat R Bhethanabotla, Subramanian K.R.S Sankaranarayanan, IEEE 2015. 
11. Evaluation of detectable depth on SH-SAW biosensor using antibody, antigen, and secondary antibody complexes, Koji Kano, Hiromi Yatsuda, Jun Kondoh, 2019 IEEE International Ultrasonics Symposium (IUS) Glasgow, Scotland.

12. Influence of the electrical extensions in AlN-BAW resonators for in-liquid biosensors, M. DeMiguel-Ramos, T. Mirea, J. Olivares, M. Clement, J. Sangrador, and E. Iborra, M. Barba, IEEE 2014.

13. Micromechanical Piezoelectric-on-Silicon BAW Resonators for Sensing in Liquid Environments, Abhinav Prasad, Ashwin A. Seshia, Jérôme Charmet, IEEE 2015.

14. H.-Y. Kim, K.-B. Kim, S. H. Cho, and Y.-I. Kim, "Analysis of resonance characteristics of Bragg reflector type film bulk acoustic resonator," Surf. Coat. Technol., vol. 211, pp. 143-147, Oct. 2012.

15. PYROLYZED CARBON BIOSENOSOR FOR APTAMER-PROTEIN INTERACTIONS USING ELECTROCHEMICAL IMPEDANCE SPECTROSCOPY, Jung A Lee, Seongpil Hwang, Kwang-Cheol Lee, Juhyoun Kwa, Se Il Par, and Seung S. Lee.

16. C-MEMS Derived Glassy Carbon Electrodes as Sensitive Electrochemical Biosensors, Bidhan Pramanick, Naresh Mandal, Debasis Mondal, Chirasree RoyChaudhuri, Suman Chakraborty.

17. DEVELOPMENT OF PATTERNABLE NANOPOROUS CARBON ELECTRODES FOR USE AS BIOSENSORS BASED ON REDOX CYCLING EFFECT, Yeongjin Lim, Deepti Sharma, and Heungjoo Shin.

18. Novel Biosensor Based on Electrospun Nanofiber and Magnetic Nanoparticles for the Detection of E. coli O157:H7, Yilun Luo, Steven Nartker, Michael Wiederoder, Hanna Miller, David Hochhalter, Lawrence T. Drzal, and Evangelyn C. Alocilja, IEEE TRANSACTIONS ON NANOTECHNOLOGY, VOL. 11, NO. 4, JULY 2012.

19. Development of MWCNT/SU-8 nanofiber composite using Electrospinning Technique for Biosensing applications, Durga Prakash M1, Siva Rama Krishna V1, Asudeb Dutta1, C.S.Sharma2 and Shiv Govind Singh1, IEEE 2014.

20. A freestanding microfluidic-based thermocouple biosensor for enzyme-catalyzed reaction analysis, Zhuqing Wang, Mitsuteru Kimura, Naoki Inomata, Takahito Ono, IEEE 2016.

21. A Thermal Biosensor Based on Enzyme Reaction, Yi-Hua Zheng, Tse-Chao Hua ,Fei Xu Engineering in Medicine and Biology 27th Annual Conference 2005.

22. A MEMS Thermal Biosensor for Metabolic Monitoring Applications, Li Wang, David M. Sipe, Yong Xu, Member, IEEE, and Qiao Lin IEEE 2008.

23. ESTIMATION OF DIAPHRAGM DEFORMATION INDUCED BY MOLECUAR ADSORPTION ON MEMS OPTICAL INTERFEROMETRIC BIOSENSOR,
Kazuhiro Takahashi, Ryo Ozawa, Makoto Ishida, Kazuaki Sawada, International Conference on Optical MEMS and Nanophotonics 2014.

24. ALL-METAL PLASMONIC METAMATERIALS FOR STRUCTURE COLOR AND AS COLORIMETRIC BIOSENSORS, Jia Zhu, Guanzhou Lin, Yun Huang, Zhuojie Chen, Xiaoyu Chen, Peimin Liu and Wengang Wu.

25. Studies on Cantilever based Triglyceride Biosensor, Renny Edwin Fernandez, Soma Sekhar B. V., Enakshi Bhattacharya, Anju Chadha, IEEE 2007.

26. A Biocompatible Affinity MEMS Sensor for Continuous Monitoring of Glucose, Xian Huangl, Siqi Li, Jerome Schultz, Qian Wang, Qiao Linl, IEEE 2009.

27. Investigation of the Scaling Rules Determining the Performance of Film Bulk Acoustic Resonators Operating as Mass Sensors Jan Weber, Mathias Link, Robert Primig, Dana Pitzer, Wolfram Wersing, and Matthias Schreiter, ieee transactions on ultrasonics, ferroelectrics, and frequency control, vol. 54, no. 2, february 2007.

28. Location-specific fabrication of suspended Metal nanowire based on electrospun nanofibers on MEMS platform. Yongkeun Oh, Dae-Sung Kwon, Wondo Kim, and Jongbaeg Kim. IEEE 2018 